\title{
A case of pemphigus vulgaris associated with ulcerative colitis
}

\author{
Joo Wan Seo ${ }^{1}$, Jongha Park ${ }^{1}$, Jin Lee ${ }^{1}$, Mi Young Kim ${ }^{1}$, Hyun Ju Choi ${ }^{1}$, Heui Jeong Jeong ${ }^{1}$, Ji Woon Lee', \\ So Young Jung', Woo Kyeong Kim ${ }^{3}$ \\ ${ }^{1}$ Division of Gastroenterology, Department of Internal Medicine, Departments of ${ }^{2}$ Dermatology, and ${ }^{3}$ Pathology, Inje University Haeundae Paik \\ Hospital, Inje University College of Medicine, Busan, Korea
}

Pemphigus vulgaris is an autoimmune bullous disorder characterized by the production of autoantibodies against the intercellular space of the epithelium. It has rarely been reported in association with inflammatory bowel disease. Ulcerative colitis is one of the forms of inflammatory bowel disease. A 62-year-old woman who had been treated for ulcerative colitis for 16 years developed pruritic bullae on the skin of her face and body. Histological findings and direct immunofluorescence examination of the skin showed pemphigus vulgaris. She was treated with systemic steroids, mesalazine, and azathioprine. Her cutaneous lesions have remained in remission and her ulcerative colitis has remained well-controlled. The relationship between pemphigus vulgaris and ulcerative colitis is unclear. An autoimmune response has been suspected in the pathogenesis of ulcerative colitis. Pemphigus vulgaris is also associated with an autoimmune mechanism. To our knowledge, this is the first case of ulcerative colitis associated with pemphigus vulgaris reported in Korea. The association may be causal. (Intest Res 2018;16:147-150)

Key Words: Colitis, ulcerative; Pemphigus; Autoimmunity

\section{INTRODUCTION}

Pemphigus is a rare, autoimmune, blistering disorder of the skin and mucosa. Pemphigus vulgaris (PV) is the most common form of pemphigus and presents as flaccid bullae of the mucous membranes and skin, caused by acantholysis. Mucous membranes are initially affected, and skin lesions develop after mucosal involvement. ${ }^{1}$

UC is one form of IBD. It is estimated that $5.2 \%$ of patients with UC have mucous membrane lesions and $11 \%$ have cutaneous lesions. ${ }^{2}$ Associations with psoriasis and lichen planus have also been reported. ${ }^{3}$ However, the association of PV and UC is rare. We describe a case of PV associated with UC.

Received October 17, 2016. Revised December 5, 2016.

Accepted January 2, 2017. Published online May 26, 2017

Correspondence to Jongha Park, Division of Gastroenterology, Department

of Internal Medicine, Inje University Haeundae Paik Hospital, Inje University

College of Medicine, 875 Haeun-daero, Haeundae-gu, Busan 48108, Korea.

Tel: +82-51-797-0200, Fax:+82-51-797-0298,E-mail: neakker@gmail.com

\section{CASE REPORT}

A 62-year-old woman presented with a 1-month history of erythematous bullae on the face, body, and both legs (Fig. 1A). She was diagnosed with UC in 1997 and prescribed sulfasalazine ( $2 \mathrm{~g}$ /day). The skin lesions were accompanied by itching. Crusts formed after scratching (Fig. 1B). When she visited the dermatology department of Inje University Haeundae Paik Hospital, a skin biopsy was performed. Histopathologic findings included the formation of clefts and vesicles containing neutrophils and eosinophils overlying basal cells (Fig. 2). Biopsy for direct immunofluorescence was obtained from the epidermis immediately adjacent to a blister. IgG and C3 deposition was identified in the intercellular spaces, compatible with PV. After the biopsy, she was treated with intravenous steroids (dexamethasone $5 \mathrm{mg}$ / day). She developed bloody stools during administration and underwent colonoscopy to determine the current status of UC. Colonoscopy revealed dirty exudate, mucosal ery-

\footnotetext{
๑ Copyright 2018. Korean Association for the Study of Intestinal Diseases. All rights reserved.

This is an Open Access article distributed under the terms of the Creative Commons Attribution Non-Commercial License (http://creativecommons.org/licenses/by-nc/4.0)

which permits unrestricted non-commercial use, distribution, and reproduction in any medium, provided the original work is properly cited.
} 

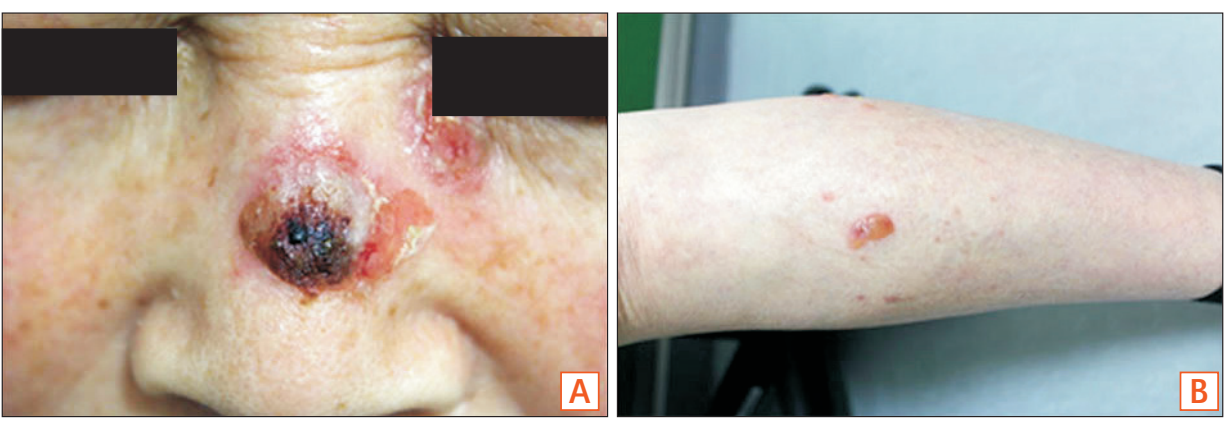

Fig. 1. Cutaneous features of the patient. (A) Crust formation on nose was appeared after scratching. (B) Pruritic erythematous bullae filled with serous exudate showed on the skin of lower leg.

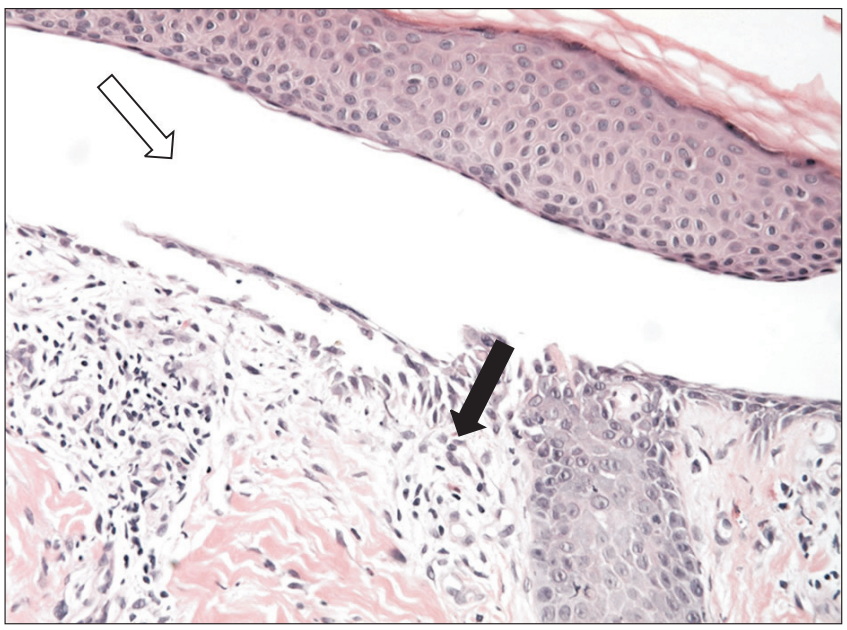

Fig. 2. Pathologic finding of the erythematous bullae of lower leg. Microscopic findings showed a suprabasilar cleft and vesicle (white arrow) with 1 to 2 layers of suprabasal keratinocytes attached to basement membrane forming part of floor of the cleft. Dermal papillae were prominent with acantholytic basal cells (black arrow) $(\mathrm{HEE}, \times 400)$.

thema, edema, and friability of the entire colon. Moreover, discrete ulcers in the descending and sigmoid colon were noted, consistent with severe and extensive UC (Fig. 3A). Sulfasalazine was discontinued. Mesalazine (6 g/day) and azathioprine (25 mg/day) were started. Her skin lesions and bloody stool improved, and she was discharged after 3 weeks. For the next 14 months, she had frequent flares of erythematous bullae and erosions on the scalp and face that required treatment with intravenous steroids.

Two years later, she underwent follow-up colonoscopy. Healing ulcers in the distal transverse colon and descending colon were observed, consistent with mild UC (Fig. 3B). Azathioprine was maintained for 19 months and then stopped due to elevated liver enzyme levels. Steroid dose was tapered and stopped after 28 months because of the improvement in cutaneous lesions. Her UC continued to be stable.

\section{DISCUSSION}

We present the case of a patient who had UC associated with PV. PV has an incidence rate between 0.1 and 0.5 per 100,000 individuals per year. The average age at onset is 40 to $60 .{ }^{4} \mathrm{PV}$ is mediated by circulating pathogenic IgG antibodies directed against the keratinocyte cell surface molecules desmoglein 3 and desmoglein 1. ${ }^{1}$ Initial symptoms are painful erosions of the oral mucosa. Skin lesions develop after mucosal involvement and are characterized by flaccid blisters and cutaneous erosions. ${ }^{5}$ Associations between PV and other autoimmune disorders such as rheumatoid arthritis, myasthenia gravis, lupus erythematous, and pernicious anemia have been reported. ${ }^{6} \mathrm{PV}$ is not known to be a skin manifestation of UC. Involvement of the gastrointestinal tract with PV is uncommon. There have been several reports of esophageal involvement with PV. However, there have been few reports on the association between PV and UC. - $^{7-10}$

In previously reported cases, 3 of 4 patients had resolution of PV after systemic steroid treatment. The other patient was treated with systemic steroid plus methotrexate, and PV resolved (Table 1). In our case, the skin lesions showed improvement after treatment with steroid and azathioprine. In the literature, systemic steroids are the mainstay of PV treatment. Azathioprine is used as an adjuvant to systemic steroid treatment. ${ }^{11}$ Unfortunately, in patients with pemphigus, the risk of steroid-induced diabetes and hyperglycemia have been reported. Our patient was diagnosed with steroidinduced diabetes during the second admission, and has been treated with insulin, metformin, and sitagliptin.

The most common reactive skin manifestations of IBD are erythema nodosum and pyoderma gangrenosum. ${ }^{12}$ Unlike these, PV is characterized by flaccid blisters and IgG antibodies against keratinocyte cell surface desmosomal molecules. In contrast, perivascular deposition of immunoglobulins and complement are seen on direct immunofluorescence in erythema nodosum, suggesting that the pathogenesis of erythema nodosum in IBD involves an immunological reaction 

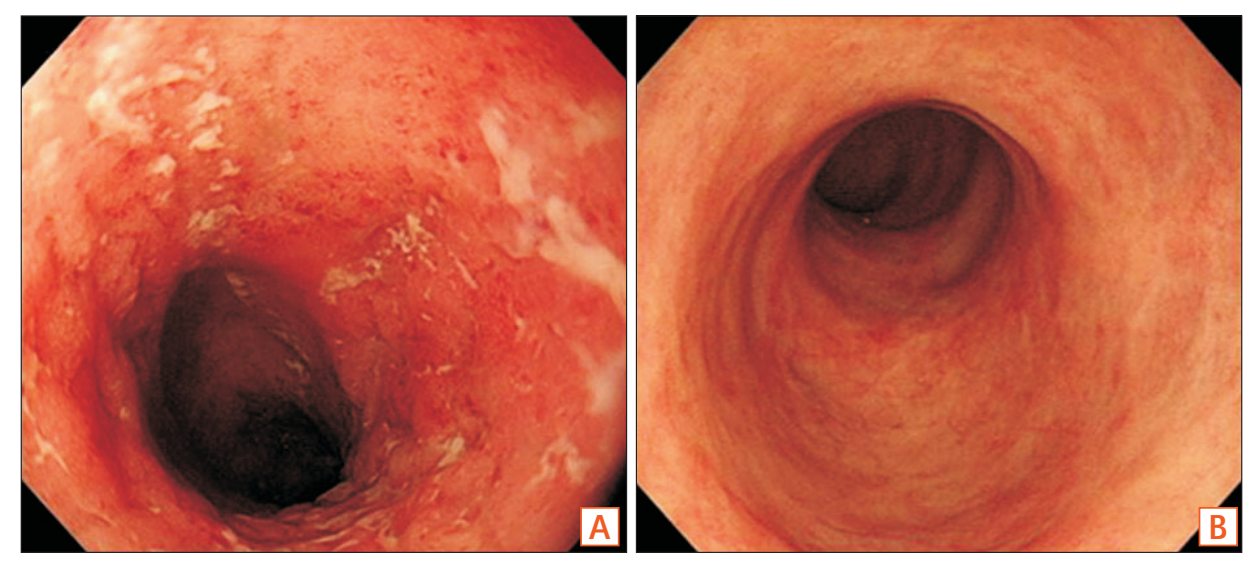

Fig. 3. Colonoscopic findings. (A) Colonoscopy showed diffuse erythema, edematous mucosa with multiple ulceration in descending colon. (B) Follow-up colonoscopy showed focal erythema and loss of vascularity.

Table 1. Clinical Features of 4 Cases of PV Associated with UC

\begin{tabular}{|c|c|c|c|c|c|}
\hline Author (year) & $\begin{array}{l}\text { Age } \\
\text { (yr) }\end{array}$ & Intraoral lesions & Skin lesions & $\begin{array}{l}\text { Presenting feature } \\
\text { of UC }\end{array}$ & $\begin{array}{l}\text { Treatment for } \\
\text { PV }\end{array}$ \\
\hline Prendiville et al. (1994) ${ }^{7}$ & 11 & $\begin{array}{l}\text { Ulcers on gingivobuccal sulcus, } \\
\text { tongue ulcer }\end{array}$ & No & Bloody diarrhea & Prednisone \\
\hline Delfino et al. $(1986)^{8}$ & 40 & Erosions on oral mucosa & Bullous lesions on the whole body & Bloody diarrhea & $\begin{array}{l}\text { Prednisone, } \\
\text { methotrexate }\end{array}$ \\
\hline Fabbri et al. $(1986)^{9}$ & 39 & Erosions on oral mucosa & Bullous lesions on the back & Bloody diarrhea & Triamcinolone \\
\hline Kacar et al. $(2002)^{10}$ & 41 & Erosion on oral mucosa & No & Rectal bleeding & Prednisolone \\
\hline
\end{tabular}

$\mathrm{PV}$, pemphigus vulgaris.

against common antigens in the bowel and skin. The pathogenesis of pyoderma gangrenosum in IBD is also considered to be an abnormal immunological response, with crossreacting autoantibodies to bowel and skin. ${ }^{13}$

However, the pathogenic relationship between PV and UC is unclear. An autoimmune mechanism is involved in the pathogenesis of PV. UC is also thought to represent an autoimmune response. A review of the literature suggests that antigens secreted by the inflamed colon cross-react with skin antigens, resulting in pemphigus. Sotiriou et al. ${ }^{14}$ suggested that inflammation of the gastrointestinal mucosa in IBD could be stimulating an immune reaction to colonic antigens, followed by cross-reactivity with antibodies to skin antigens. Nico et al. ${ }^{15}$ suggested that exposure of colonic mucosal antigens to the immune system has the potential to stimulate anti-epithelial autoimmunity, resulting in pemphigus. Some reports suggested that colectomy plays a therapeutic role in patients with UC associated with immunobullous skin disease. Sotiriou et al. ${ }^{14}$ reported that skin disease resolved in a patient who had linear IgA bullous dermatosis and UC, while another patient who had not undergone colectomy needed to continue treatment due to active skin disease. Three additional cases showed resolution of skin disease after proctocolectomy. ${ }^{16}$ This suggests that inflamed bowel in UC is a source of antigenic stimulation, causing immunobullous skin disease.

Moreover, there is some speculation that the association between UC and immune-mediated mucocutaneous blistering disorders may be triggered by an epitope spreading mechanism. ${ }^{17,18}$ A study conducted in the United Kingdom revealed that patients with linear IgA bullous dermatosis had a higher prevalence rate of UC. ${ }^{19}$ Uchiyama et al. ${ }^{20}$ reported a case of IgA/IgG pemphigus combined with UC. They suggested that IgA autoantibodies might be induced by colonic inflammation. Epitope spreading may explain the association between UC and PV. In our case, UC appeared first, followed by PV, supporting the concept that inflammation of the colon is the inciting factor.

This is the first case of UC associated with PV reported in Korea. This case highlights the possible causal association between UC and PV. PV may be an extraintestinal dermatologic manifestation of UC.

\section{FINANCIAL SUPPORT}

The authors received no financial support for the research, 
authorship, and/or publication of this article.

\section{CONFLICT OF INTEREST}

No potential conflict of interest relevant to this article was reported.

\section{AUTHOR CONTRIBUTION}

J.W.S. wrote the initial draft which was then extensively edited by J.P. All authors were involved in critical revision of the manuscript and have approved the final manuscript for submission.

\section{REFERENCES}

1. Mihai S, Sitaru C. Immunopathology and molecular diagnosis of autoimmune bullous diseases. J Cell Mol Med 2007;11:462-481.

2. Areias E, Garcia e Silva L. Cutaneous manifestations of ulcerative colitis. Med Cutan Ibero Lat Am 1987;15:185-197.

3. Timani S, Mutasim DF. Skin manifestations of inflammatory bowel disease. Clin Dermatol 2008;26:265-273.

4. Kneisel A, Hertl M. Autoimmune bullous skin diseases. Part 1: clinical manifestations. J Dtsch Dermatol Ges 2011;9:844-856.

5. Amagai M. Pemphigus vulgaris. In: Bolognia J, Jorizzo, J, Schaffer J, eds. Dermatology. Volume 1. 3rd ed. London: Elsevier, 2012:461-474.

6. Pemphigus: current concepts. Ann Intern Med 1980;92:396405.

7. Prendiville JS, Israel DM, Wood WS, Dimmick JE. Oral pemphigus vulgaris associated with inflammatory bowel disease and herpetic gingivostomatitis in an 11-year-old girl. Pediatr Dermatol 1994;11:145-150.

8. Delfino M, Suppa F, Piccirillo A. Pemphigus vulgaris and ulcerative colitis. Dermatologica 1986;172:230.
9. Fabbri P, Emmi L, Vignoli L, et al. Chronic pemphigus vulgaris associated with ulcerative rectocolitis: apropos of a clinical case. G Ital Dermatol Venereol 1986;121:355-359.

10. Kacar S, Sezgin O, Sahin T. Pemphigus vulgaris and ulcerative colitis. Am J Gastroenterol 2002;97:507-508.

11. Chams-Davatchi C, Esmaili N, Daneshpazhooh M, et al. Randomized controlled open-label trial of four treatment regimens for pemphigus vulgaris. J Am Acad Dermatol 2007;57:622-628.

12. Levine JS, Burakoff R. Extraintestinal manifestations of inflammatory bowel disease. Gastroenterol Hepatol (N Y) 2011;7:235241.

13. Huang BL, Chandra S, Shih DQ. Skin manifestations of inflammatory bowel disease. Front Physiol 2012;3:13.

14. Sotiriou MC, Foo CW, Scholes CT, Zone JJ. Immunobullous disease and ulcerative colitis: a case series of six patients. Br J Dermatol 2015;173:792-796.

15. Nico MM, Hussein TP, Aoki V, Lourenço SV. Pyostomatitis vegetans and its relation to inflammatory bowel disease, pyoderma gangrenosum, pyodermatitis vegetans, and pemphigus. J Oral Pathol Med 2012;41:584-588.

16. Watchorn RE, Ma S, Gulmann C, Keogan M, O'Kane M. Linear IgA disease associated with ulcerative colitis: the role of surgery. Clin Exp Dermatol 2014;39:327-329.

17. Chan LS, Vanderlugt CJ, Hashimoto T, et al. Epitope spreading: lessons from autoimmune skin diseases. J Invest Dermatol 1998;110:103-109.

18. Sachsenberg-Studer EM, Runne U, Wehrmann T, et al. Bullous colon lesions in a patient with bullous pemphigoid. Gastrointest Endosc 2001;54:104-108.

19. Paige DG, Leonard JN, Wojnarowska F, Fry L. Linear IgA disease and ulcerative colitis. Br J Dermatol 1997;136:779-782.

20. Uchiyama R, Ishii N, Arakura F, et al. IgA/IgG pemphigus with infiltration of neutrophils and eosinophils in an ulcerative colitis patient. Acta Derm Venereol 2014;94:737-738. 